\title{
Thyrotropinoma - not just hyperthyroidism
}

\author{
Edyta Gurgul, Maria Gryczynska, Adam Maciejewski, Aleksandra Klimowicz, \\ Dagny Lapinska, Marek Ruchala
}

Department of Endocrinology, Metabolism and Internal Medicine Poznan University of Medical Sciences, Poland

Thyrotropinoma is a very rare cause of hyperthyroidism (pituitary tumor that secrets thyrotropin).

In October 2014 a 41 -year-old male patient was hospitalized due to anaemia and diarrhea.

Abdomen CT scan, gastroscopy, colonoscopy and parasite tests did not reveal any abnormalities. The patient had lost $18 \mathrm{~kg} 7$ years ago and his weight had been stable since then (BMI 21,3kg/ $\mathrm{m}^{2}$ ). He has no children (he got married 7 years ago).

Diarrhea resolved on gluten-free diet (suspected coeliac disease).

ASCA antibodies were positive (suspected Crohn disease).

The gastroenterological diagnosis has not been clearly stated.

The patient was referred to our department. He presented with diarrhea, velvet skin, loss of facial, chest, axillary and pubic hair; he admitted occasional heart palpitations.

The laboratory results were: TSH 13,56uIU/ml $[0,27-4,20]$ (no increase in TRH stimulation test) fT3 12,57 pmol $/ 1[3,90-6,70]$, fT 4 55,55pmol/1 [11,5 - 21,0], low FSH, LH, DHEA-S, testosterone, GH and IGF-1.

The results of insulin tolerance test showed secondary adrenal insufficiency.

Pituitary MRI demonstrated a solid intra- and suprasellar pituitary macroadenoma $(25 \mathrm{~mm})$ with sphenoid and cavernous sinus invasion and optic chiasm dislocation.

Thyroid ultrasound showed low echogenicity of thyroid gland with no focal lesions.

Densitometry revealed osteoporosis.

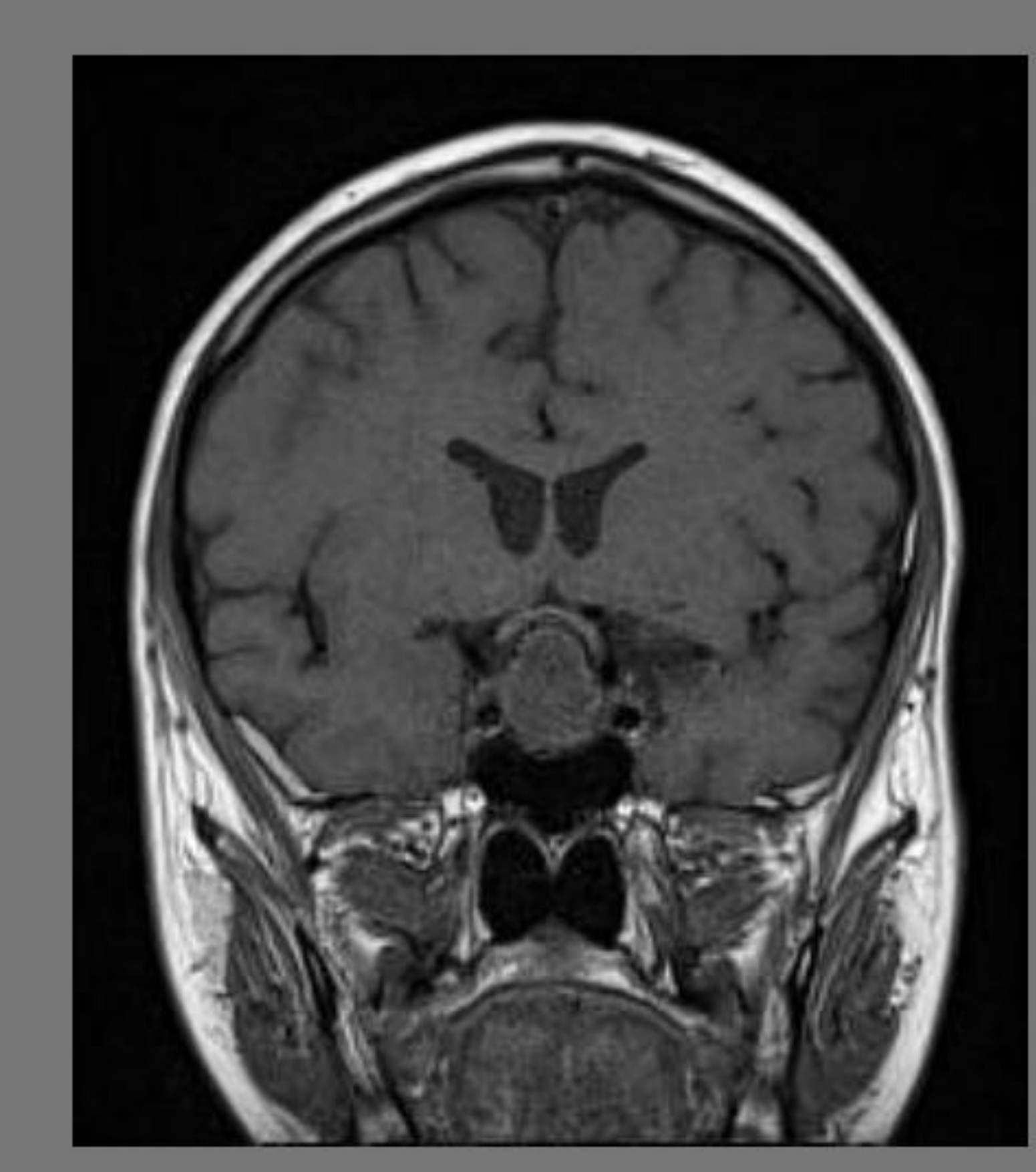

The treatment was discussed with neurosurgons. We introduced octreotide injections every 4 weeks, hormonal replacement and osteoporosis therapy.

Control pituitary MRI performed in February 2015 revealed no progression of pituitary macroadenoma. The patient presented a significant clinical improvent. TSH, fT3 and fT4 levels still remained high. We decided to continue octreotide therapy.

In April 2015 the patient presented with psychiatric disorders (aggression, cognitive impairment). No significant abnormalities were found in biochemical results or in neurological examination.

MRI showed stable pituitary tumor with persistent optic chiasm dislocation.

The patient has been qualified for neurosurgical treatment.

We conclude that clinical image of thyrotropinoma may be confusing due to coincident symptoms of pituitary deficiency and potential concomitant diseases. Optimal diagnosis and treatment require a multidisciplinary approach. 